\title{
OPTIMAL ARRAY COMBINER FOR SEQUENCE DETECTORS
}

\author{
Miguel A. Lagunas, Ana I. Perez-Neira, Josep Vidal \\ TSC Departament, Modulo D5 \\ Campus Nord UPCc/ Gran Capita s/n \\ 08034 Barcelona- SPAIN
}

\begin{abstract}
The use of spatial diversity at the receiver frontend together with a sequence detector implies a joint design problem of the spatial combiner and the sequence detector impulse response. This joint design is usually faced under the constraint that the impulse response of the sequence detector is matched to the channel plus combiner response. This procedure maximizes the signal to noise ratio at the input of the detector but does not guarantee that the so-called effective signal to noise ratio is maximized. This work presents a procedure that, starting from the matched criteria, faces directly the maximization of the effective signal to noise ratio, yet preserving all the features of the spatial processor in terms of co-channel and high order intersymbol interference rejection.
\end{abstract}

\section{INTRODUCTION}

The increasing traffic demands, imposed by a large number of users in a single cell, in mobile communications move to manufacturers to seek for the potential of spatial diversity techniques to alleviate such congestion problems. On the other hand, base-stations for the mobile are sophisticated systems, both in prize technology and operation, that impose constraints on the use of spatial diversity being crucial that the spatial processor is designed jointly with the rest of the subsystems forming the baseband processor. In this respect, matched filtering and the sequence detector are unavoidable subsystems that have to interact constructively with the spatial processor in order to improve the global performance of the receiver.

To specify the relationship between the spatial combiner and the temporal processor, namely the matched filter and the sequence detector, it is necessary to assign the appropriate role to each part. The sequence detector, normally a Viterbi Equalizer (VE) is the optimum procedure to combat the Inter Symbol Interference (ISI); but it is quite sensitive to co-channel interferers or temporal correlated noise [1]. At the same time, the VE is based in a metric computation measuring the distance of the received sample with the product of a candidate sequence by the impulse response of the communication channel, which is denoted by the Desired Impulse
Response (DIR). The major impact of the DIR length is both in complexity and decoder delay, since the number of candidate sequences increases with the DIR length. In summary, any pre-processing aims the reduction of the DIR's length and to remove co-channel interferers. One attempt to reduce the length of the DIR was reported in the seventies for voice band data transmission [6]. The alternative, still in use, was to set a pre-equalizer, named as Forward Equalizer, which major objective was to reduce the length of the DIR. The drawbacks of the FE are twofold; first, it do not help on interference removal; second, it introduces temporal correlation in the noise. Both effects have a negative impact in the formulation of the ML sequence detector and in the performance of the baseline VE [5].

The spatial processor removes the two difficulties of the FE, namely it does not introduces temporal correlation in the noise and it is able to remove co-channel interferers being quite effective in this role. Furthermore, under a training sequence it is capable of removing late arrivals with otherwise increase the ISI order and, as a consequence the length of the DIR. This summarizes the role of the spatial processor in a single task which is to remove, from the combiner output, everything which is not motivated by the candidate sequence plus white gaussian noise. With respect the matched filter, we will consider the case of a broadband combiner which is equivalent to include a matched filter for every diversity channel. The scheme of this combiner is depicted in Figure 1.

The broadband combiner is mandatory for the cases where either the size of the aperture or the channel spread is high enough to reduce severely the coherence among the diversity channels. When this is not the case, a single matched filter is the optimum receiver and the combiner reduces to a narrowband one. Since our presentation is valid for the two cases, we will preserve the broadband architecture. In [2] it is shown a procedure to reduce a broadband design to a narrowband design based in a rank-one approximation of the first. The joint design, under the premises described before, was reported in [2][3]. Also the basic concepts involved in these works can be found in [4][5][6] as a FE design problem both for sequence detectors and for Decision Directed equalizers. 


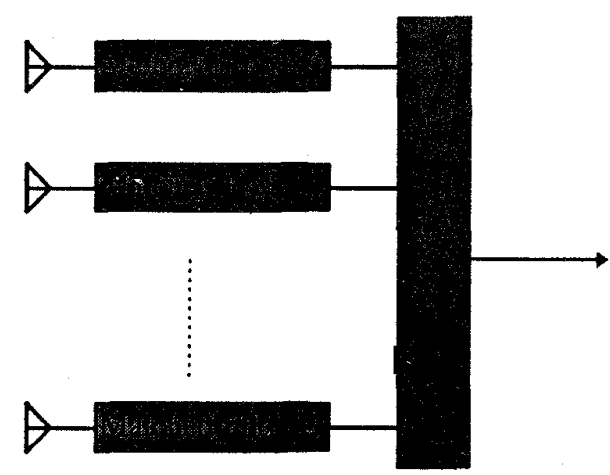

Figure 1. The broadband spatial combiner, including a matched filter for every diversity channel.

This work will present a refinement of the socalled Matched DIR (MDIR) procedure [2], which consist in the direct maximization of the Effective Signal to Noise Ratio (SNRe) of the sequence detector. Since MDIR is based on the SNR at the input of the sequence detector it does not ensures that it is working under optimum conditions. Since the sequence detector is controlled by the ratio between minimum distance between candidate sequences and the gaussian noise power, the procedure to be reported hereafter focus on directly the SNRe and, as a consequence, produces always better performance than the MDIR design. The reason is that only the SNRe acts in controlling the Bit Error Rate (BER) bound. The resulting algorithm improves the SNRe, yet preserving the advantages and features of the MDIR design. The complexity associated with the algorithm is very low and do not represent any problem to its direct implementation in the baseband processor. Starting from the MDIR design, the DIR is iteratively changed until is minimum distance to any error sequence, divided by the noise power, is maximized. The procedure will be named Minimum Distance (MDIS) and will be described in section III, right after and introduction in section II of the MDIR method.

\section{THE MDIR METHOD}

The joint design of the spatial combiner $\underline{b}$ and the DIR $\underline{h}_{0}$ of the sequence detector is based on the minimization of the Mean Square Error $\eta$,

$\mathrm{E}\left(/ \mathrm{e}(\mathrm{n}) /^{2}\right)=\mathrm{E}\left(/ \underline{\mathrm{b}}^{\mathrm{H}} \cdot \underline{\mathrm{X}}_{\mathrm{n}}-\underline{\mathrm{h}}_{\mathbf{0}}{ }^{\mathrm{H}} \cdot \underline{\mathrm{d}}^{\mathcal{R}}\right)=\eta$

where $\underline{X}_{n}$ is the snapshot from the spatial aperture and $\underline{d n}$ is a vector, with equal length of the DIR, containing successive symbols of a given training sequence. Since the presence of channel coding does not modifies our results, we will assume that the symbols of the training sequence are uncorrelated, i.e. $E\left(\underline{d}_{n} \cdot \underline{d}_{n}{ }^{H}\right)$ is equal to the identity matrix. A scheme of the temporal processor is depicted in Figure 2. Perfect synchronism is assumed at the symbol sampler in our presentation. The training sequences are formed, as in the GSM standard [7], by 26 symbols located in the middle of 256 information symbols frame.

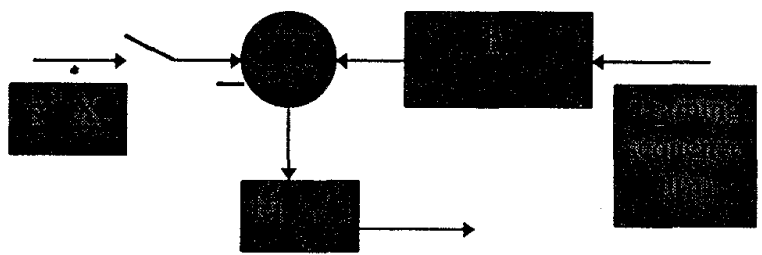

Figure 2. The MSE defined from the difference between the output of the spatial processor, sampled at the symbol rate, and the channel response, represented by the DIR filtering the training sequence symbols.

In selecting a constraint for the joint minimization problem, in order to avoid the trivial solution, there are several choices [5]. Nevertheless, the most popular is based in the control of the energy of the part of $y(n)$ that corresponds to the symbols included in the training sequence; note that these symbols will not cause any detector problem, because they are optimally combined by the DIR. To formulate the constraint, let us assume that the received snapshot is formed by two terms; the first term is formed by the multichannel response to the symbols included in the sequence detector and a second term which includes late multipath arrivals, interferences and noise.

$$
\underline{\mathrm{X}}_{\mathrm{n}}=\mathbf{G} \cdot \underline{\mathrm{d}}_{\mathrm{n}}+\underline{\mathrm{X}}_{\mathrm{on}}
$$

The signal part of the combiner output will be provided only by the first term and its energy will be (3), already constrained to one.

$$
\underline{b}^{\mathbf{H}} \cdot \mathbf{G} \cdot \mathbf{G}^{\mathrm{H}} \cdot \underline{\mathrm{b}}=\mathbf{1}
$$

When this constraint is used for the minimization of the MSE defined in (1), the resulting $\underline{b}$ and $\underline{h}_{0}$ maximize the signal to noise ratio at the input of the sequence detector defined as (4); where $\mathbf{R}_{\mathrm{o}}$ is the covariance matrix of $\underline{X}_{\text {on }}$.

$\operatorname{SNR}=\left(\underline{b}^{\mathrm{H}} \cdot \mathbf{G} \cdot \mathbf{G}^{\mathrm{H}} \cdot \underline{\mathbf{b}}\right) /\left(\underline{\underline{b}}^{\mathrm{H}} \cdot \mathbf{R}_{\mathrm{o}} \cdot \underline{\mathrm{b}}\right)$

The estimation of the channel matrix $\mathbf{G}$ and the covariance matrix $\mathbf{R}_{\mathrm{o}}$, which are the basis of a vector $M L$ formulation of the detector problem, are defined as (5) and can be estimated during the presence of the training frame.

$$
G=E\left(\underline{X}_{n} \cdot \underline{d}_{n}^{H}\right)
$$




$$
\begin{aligned}
& \mathbf{R}=\mathrm{E}\left(\underline{\mathrm{X}}_{n} \cdot \underline{\mathrm{X}}_{n}\right) \\
& \mathbf{R}_{\mathrm{o}}=\mathbf{R}-\mathbf{G} \cdot \mathbf{G}^{\mathrm{H}}
\end{aligned}
$$

Going back to the constrained minimization problem, it is easy to find that the optimum combiner is given by the eigenvector associated to the minimum eigenvalue of (6.a) and the optimum DIR is given by (6.b). The maximum of the SNR is given by the inverse of the eigenvalue minus one.

$$
\begin{aligned}
& R \cdot \underline{b}=\lambda \cdot \mathbf{G} \cdot \mathbf{G}^{\mathrm{H}} \cdot \underline{\mathrm{b}} \\
& \underline{\mathrm{h}}_{\mathrm{o}}=\mathbf{G}^{\mathrm{H}} \cdot \underline{\mathrm{b}}
\end{aligned}
$$

It is just the last equation the responsible for the MDIR name to the procedure, since the DIR $\underline{h}_{0}$ is matched to the response of the communication channel plus the spatial combiner when signal vector $\underline{d}_{n}$ has been transmitted.

\section{MINIMUM DISTANCE METHOD}

The signal to noise ratio defined for the MDIR design is just a measure of how good is, with respect the noise, the signal plus ISI at the input of the sequence detector. In other words, this SNR does not tell us how difficult it could be for the sequence detector to combat the residual ISI. When a information frame is under processing, the DIR is matched as (6.b) and no interferers or late arrivals are present at the combiner output; the input of the equence detector is gaussian distributed with power equal to the MSE $\eta$, and mean equal to $\underline{h}_{0}{ }^{H}$. ${ }_{\text {. }}$. Thus the metric to be computed by the sequence detector is the square of the difference between the received signal and the mentioned mean. An error deciding the optimum sequence will be produced, with the highest probability, to the closest vector d'; in consequence a bound of the BER will be given by the minimum distance between two valid sequences in the detection space. In summary, the socalled effective SNRe is defined as (7),

$$
\operatorname{SNRe}=\min . / \underline{h}^{\mathrm{H}} \cdot \underline{\varepsilon}_{j} / /(4 . \eta)
$$

where min. stands for the most dangerous error sequence $\underline{\varepsilon}_{\mathrm{j}}$ (i.e. the difference of two valid $\underline{\mathrm{dn}}$ ) which minimizes the numerator. The BER is bounded as (8)

$$
\mathrm{BER}<=\mathrm{Ko} \cdot \mathrm{Q}\left(\mathrm{SNRe}^{1 / 2}\right)
$$

where Ko depends on the probability of the error sequence and $Q($.$) is the probability error function.$

Being our objective to increase the SNRe, the algorithm will introduce a small perturbation in the DIR. Since this perturbation has to increase the numerator, the perturbation is chosen in the direction of the worst error sequence

$$
\underline{h}_{0}=\underline{h}_{0}+\mu \cdot \underline{\varepsilon}_{j} \cdot \operatorname{sign}\left(\operatorname{Re}\left(\underline{h}_{o}^{H} \cdot \underline{\varepsilon}_{j}\right)\right)(9)
$$

where sign(.) stands for the sign function and $\mathrm{Re}$ for the real part. This perturbation avoids the use of the dot product because it is assumed that the error sequence has zero quadrature component as corresponds with a BPSK modulation. In other words, the DIR is only in-phase perturbed leaving constant its quadrature components. Alternatives to this algorithm can be found in [10] in the framework of linear discriminants for pattern recognition purposes.

Every perturbation introduced in the DIR causes an increase in the MSE. Since the metric is valid whenever the DIR remains matched to the combiner response, the following constraint has to be set for the new combiner,

$$
\mathbf{G}^{\mathrm{h}} \cdot \underline{\mathrm{b}}=\underline{\mathrm{h}}_{\mathrm{o}}
$$

with respect to the new MSE, since it remains with the same formulation $\underline{b}^{h} \cdot R_{0} \cdot \underline{b}$, it is clear that the optimum combiner, after perturbation, is:

$$
\underline{b}=\mathbf{R}_{\mathbf{o}}^{-1} \cdot \mathbf{G} \cdot\left(\mathbf{G}^{\mathrm{H}} \cdot \mathbf{R}_{\mathbf{o}}^{-1} \cdot \mathbf{G}\right)^{-1} \cdot \underline{\mathbf{h}}_{\mathrm{o}}
$$

and the corresponding MSE,

$$
\eta=\underline{h}_{0}^{H} \cdot\left(G \cdot R_{0}^{-1} \cdot G^{H}\right)^{-1} \cdot \underline{h}_{0}
$$

The perturbations continue until the SNRe increases. The procedure cannot be carried over in a single iteration since the target error sequence of the SNRe uses to change after a few iterations. It is important to note that the major problem of the procedure is the presence of strong interferers because, in such a case, the MSE will show a significative increment, even for low perturbations. There are two problems associated with the strong interferences; first, small perturbations do not increase the SNRe; and, the increase of interferers power at the combiner output promotes that the metric used in the sequence detector is not longer in correspondence with the exact likelihood of the candidates sequences.

\section{PERFORMANCE EVALUATION}

In order to evaluate the performance of the procedure, the following scenario was prepared: The array was a five sensor ULA array and the length of the sequence detector was set to four; the desired source was located at the array broadside with variable $\mathrm{C} / \mathrm{N}$ ranging from -4 up to $14 \mathrm{~dB}$; the ISI was set of order 3 (actual DIR of four taps) with DOAs equal to $10,-7$ and 40 degrees; the coefficients of these rays were set to $0.7,-0.6$ and 0.3 with respect the direct path at the broadside. 
Figure 3 shows above the gain of $\mathrm{SNRe}$ in $\mathrm{dB}$ of the MDIS versus the corresponding MDIR (top) and the loss observed in the SNR, also in $\mathrm{dB}$. Regardless the target SNR is the effective one, it is evident that a gain of $4 \mathrm{~dB}$ is maintained in all the range of desired $\mathrm{C} / \mathrm{N}$ (carrier to noise ratio).

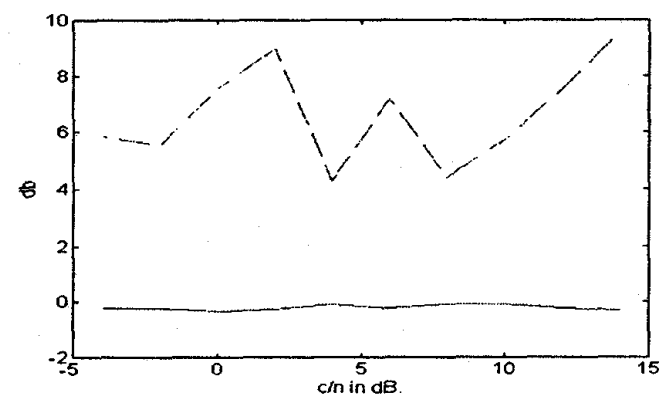

Figure 3. SNRe improvement for MDIS versus MDIR for different $\mathrm{C} / \mathrm{N}$ of the desired (Top). Loss in the input SNR to the sequence detector of MDIS versus MDIR (Bottom).

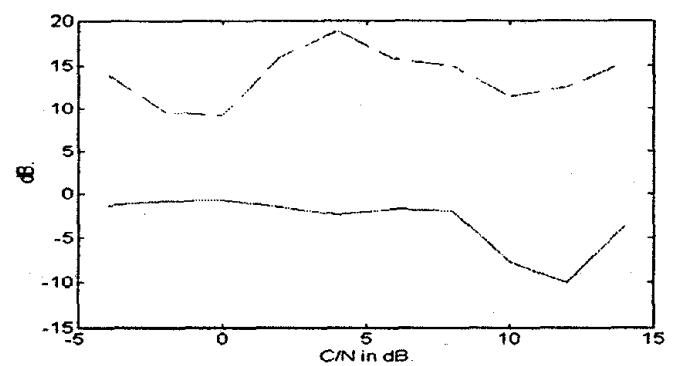

Figure 4. The same as Fig. 3 including a late arrival in the signal scenario

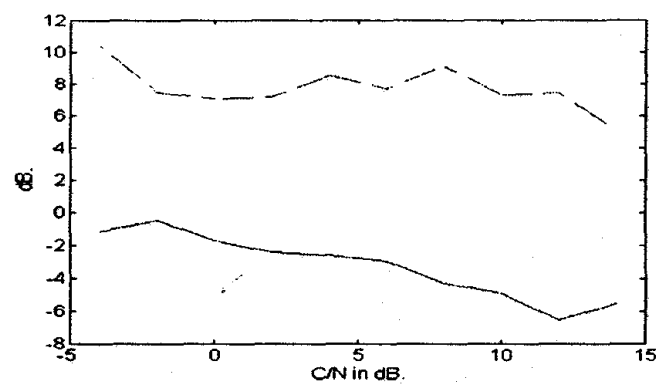

Figure 5. The same as Fig 4 when the scenario includes a co-channel interference.

It is important to note that regardless the SNR is not the crucial parameter, the amount of SNRe gained by MDIS is due with a small loss in the input SNR. The second scenario is the same that in Figure 3, but adding a late arrival ( five symbol intervals delay), impinging the aperture from $-30^{\circ}$ and with a coefficient, respect to the desired, equal to -0.9 . Figure 4 shows the gain and loss respectively for this new scenario. Clearly the MDIS still is always superior in terms of the SNRe. Finally, Figure 5 shows the same performance plots for the case when an interference, $\mathrm{C} / \mathrm{I}$ equal to $-10 \mathrm{~dB}$ and $\mathrm{DOA}$ equal to $20^{\circ}$ was added to the scenario of Fig. 4. Again the improvement obtained from MDIS, versus MDIR, is quite evident

\section{REFERENCES}

[1] P.D. Shaft, E.C. Kather. " Experimental measurement of Viterbi decoding in burst channels". IEEE Trans. on Communications, Vol. COM-27, no. 9, pp.1360-1366, Aug. 1978.

[2] M.A.Lagunas, A.I. Perez, J. Vidal. " Joint beamforming and Viterbi equalizers in wireless communications". To appear in Proc. Asilomar. 1977.

[3] F. Pipon, P. Chevalier, P. Vila, J.J. Monott. "Joint spatial and temporal equalization for channels with ISI and ICI: Theoretical and Experimental results for a basestation". Proc. IEEE Workshop on Signal Advances in Wireless Communications, SPAWC, Paris, pp 309-312, April 1997.

[4] Falconer, F.R. Magee. "Adaptive channel memory truncation for maximum likelihood sequence estimation" Bell Sys. Tech. J., Vol. 9, pp. 1541-1562, Nov. 1973.

[5] F.R. Magee. "A comparison of compromise Viterbi algorithm and standard equalization techniques over band limited channels". IEEE Trans. on Communications, Vol. COM-23, no. 3, pp. 361-367, March 1975.

[6] D.R. Falconer, F.R. Magee. "Evaluation of decision feedback equalization and Viterbi algorithm detector for voice band data transmission". IEEE Trans. on Communications, Vol. COM-24, no. 10, pp. 1130-1139, October 1976.

[7] R. Steele. "Mobile Radio Communications". IEEE Press, Pentech Press, pp. 723-732. 1992.

[8] European Telecommunications Standard, ETS 300574, II Edition, Source ETSI TC-SM6, Page 12, Aug. 1975.

[9] C.T. Beare. "The choice of the desired impulse response in combined linear Viterbi algorithm equalizers". IEEE Trans. on Communications, Vol. COM-26, no. 8, pp. 1301-1307, Aug. 1978.

[10] R.O. Duda, P. Hart. "Pattern recognition and scene analysis". Chapter 5, pp. 130-186, Wiley, NY 1973.

This work has been supported by CICYT of Spain and CIRIT of Catalunya 